\title{
Fractional Order Two Temperature Thermo-Elastic Behavior of Piezoelectric Materials
}

\author{
Essam Bassiouny ${ }^{1,2}$, Refaat Sabry ${ }^{1,3,4}$ \\ ${ }^{1}$ Department of Mathematics, Faculty of Science and Humanitarian Studies, \\ Salman Bin AbdulAziz University, Al Kharj, KSA \\ ${ }^{2}$ Department of Mathematics, Faculty of Science, Fayoum University, Fayoum, Egypt \\ ${ }^{3}$ Theoretical Physics Group, Department of Physics, Faculty of Science, Mansoura University, \\ Damietta Branch, New Damietta, Egypt \\ ${ }^{4}$ International Centre for Advanced Studies in Physical Sciences, Faculty of Physics and Astronomy, \\ Ruhr University Bochum, Bochum, Germany \\ Email: esambassiouny@yahoo.com, Sabry_nonlinear@yahoo.com
}

Received July 13, 2013; revised August 21, 2013; accepted August 27, 2013

Copyright (C) 2013 Essam Bassiouny, Refaat Sabry. This is an open access article distributed under the Creative Commons Attribution License, which permits unrestricted use, distribution, and reproduction in any medium, provided the original work is properly cited.

\begin{abstract}
A new mathematical model of time fractional order heat equation and fractional order boundary condition have been constructed in the context of the generalized theory of thermo piezoelasticity. The governing equations have been applied to a semi infinite piezoelectric slab. The Laplace transform technique is used to remove the time-dependent terms in the governing differential equations and the boundary condition. The solution of the problem is first obtained in the Laplace transform domain. Furthermore, a complex inversion formula of the transform based on a Fourier expansion is used to get the numerical solutions of the field equations which are represented graphically.
\end{abstract}

Keywords: Fractional Order; Two Temperature; Generalized Thermoelectricity; Weak Diffusion; Thermal Loading; Piezoelectric Materials; Ceramics

\section{Introduction}

Chen and Gurtin [1-3] have formulated a theory of heat conduction in deformable bodies, which depends upon two distinct temperatures, the conductive temperature $\varphi$ and the dynamical temperature T. Regarding time independent situations, the difference between these two temperatures is proportional to the heat supply. However, in the absence of any heat supply, the two temperatures are identical $[1,2]$. On the other hand in time dependent problems, particularly for wave propagation problems, the two temperatures are generally different regardless of the presence of heat supply. The two temperatures T, $\varphi$ and the strain are found to have representations in the form of a traveling wave plus a response, which occur instantaneously throughout the body [4]. Warren and Chen [5] investigated the wave propagation in the twotemperature theory of thermoelasticity, but Youssef [7] investigated this theory in the context of generalized thermoelasticity.

Because the non-local property of fractional order of differential equations (FODE), FODE becomes prominent and extensively used in many applications in fluid mechanics, physics, engineering, viscoelasticity and many other fields. The presence of the fractional order operator in the differential equations affects the history of the system, which means that the next states of the system will depend, on the current state and also upon all of its previous states, making it more realistic: Caputo [7], Mainardi [8] and Podlubny [9]. FODE has been used successfully in modeling of various physical phenomena and in many applications such as chemistry, biology, electronic, wave propagation and viscoelasticity Hilfer [10], Caputo and Mainardi [11], Caputo [12], Bagley and Torvik [13], Koeller [14] and Rossikhin and Shitikova [15].

In the second half of the $19^{\text {th }}$ century, both of the theory of fractional derivatives and integrals were established. The first application of fractional derivatives was applied by Abel to solve an integral equation that arises in the formulation of the Tautochrone problem $[16,17]$.

Various definitions and approaches of fractional derivatives have become the main purpose of many studies 
$[18,19]$.

Kimmich [19] study time — fractional diffusion — wave equation and use the Riemann-Liouville fractional integral as follows:

$$
I^{\xi} f(t)=\left\{\begin{array}{lc}
\frac{1}{\Gamma(\xi)} \int_{0}^{t}(t-\tau)^{\xi-1} f(\tau) \mathrm{d} \tau, & 0<\xi \leq 2, \\
f(t), & \xi=0
\end{array}\right.
$$

where $\Gamma(\xi)$ is the Gamma function.

Fujita $[20,21]$ considered a fractional order heat wave equation for the case $1 \leq \xi \leq 2$ obtained from the non local constitutive equation for the heat flux components $q_{i}$ in the form

$$
q_{i}=-\kappa I^{\xi-1} T_{i}, \quad 1<\xi \leq 2
$$

Povestenko [22,23] used the heat Equation (2) to study the theories of thermal stresses based on space-time fractional telegraph equations.

To eliminate the paradox of the instantaneous propagation of heat, Cattaneo [24] introduced a law of heat conduction to replace the classical Fourier law of heat conduction. The propagation of discontinuities of solutions in this theory was investigated by Ezzatt and Karamany [25].

Ezzatt and Karamany [25-28] established a new model of fractional heat equation based on a Taylor expansion of time-fractional order. They studied the non-homogeneous anisotropic elastic solid using two new models and they considered the uniqueness theorem in linear fractional two temperatures thermoelasticity and the theory of a perfect conducting thermoelastic medium. Also, they constructed a new model of electro-thermoelasticity in the context of a new consideration of heat conduction with fractional order.

Sherief et al. [16] used the following form of the heat conduction law

$$
q_{i}+\tau_{o} \frac{\partial^{\xi} q_{i}}{\partial t^{\xi}}=-\kappa \frac{\partial T}{\partial t}, \quad 0<\xi \leq 1
$$

and derived the governing equations of the fractional order theory of thermoelasticity using Caputo [7] definition of fractional derivatives of order $0 \leq \xi \leq 1$ of absolutely continuous function $f(t)$ given by:

$$
\begin{gathered}
\frac{\mathrm{d}^{\xi}}{\mathrm{d} t^{\xi}} f(t)=I^{1-\xi} f^{\prime}(t) \\
I^{\xi} f(t)=\int_{0}^{t} \frac{(t-s)^{\xi-1}}{\Gamma(\xi)} f(s) \mathrm{d} s
\end{gathered}
$$

where $I^{\xi}$ is the fractional integral of the function $f(t)$ of order $\xi$ defined by [19]. In the limit as $\xi \rightarrow 1$, Equation (3) can be reduced to Cattaneo law [24].

A new formula of heat conduction has been considered in the context of fractional integral operator defined by Youssef [29] who introduces the following form of heat conduction law

$$
q_{i}+\tau_{o} \dot{q}_{i}=-\kappa I^{\xi-1} T_{i}, 0<\xi \leq 2
$$

Taking into consideration the works of Fujita $[20,21]$ and Povestenko [22,23], Youssef proved the uniqueness of the solutions in this case.

In the present work a model for generalized thermopiezoelasticity has been constructed in the context of the fractional heat equation where $0<\xi<2$ to describe different types of diffusion where $0<\xi<1$ corresponds to weak diffusion, $\xi=1$ corresponds to normal diffusion, $1<\xi<2$ corresponds to strong diffusion and $\xi=2$ corresponds to ballistic diffusion. This is used to investigate the propagation of thermal wave through a semi infinite slab subjected to thermal loading of fractional order of exponential type applied for finite period of time.

\section{Governing Equations}

In the absence of body force, free charge and inner heat sources, we consider generalized thermo-piezoelectric governing differential equations Youssef [29] and Youssef and Bassiouny [30] follows:

Equations of motion:

$$
\sigma_{i j, j}=\rho \frac{\partial^{2} u_{i}}{\partial t^{2}}
$$

Equation of entropy increment (in the absence of inner heat source):

$$
q_{i, i}=-T_{o} \frac{\partial \eta}{\partial t},
$$

Stress-strain-temperature:

$$
\sigma_{i j}=c_{i j k l} e_{k l}-h_{k i j} D_{k}-\beta_{i j} \theta,
$$

Gauss equation and electric field relation:

$$
D_{i, i}=0
$$

$$
\begin{aligned}
& E_{i}=-v_{i}, \\
& E_{i}=h_{i k l} e_{k l}+\tau_{i k} D_{k}-d_{i} \theta
\end{aligned}
$$

Equation of entropy density:

$$
\eta=\beta_{i j} e_{i j}+d_{i} D_{i}+c T
$$

Strain-displacement relations:

$$
e_{i j}=\frac{1}{2}\left(u_{i, j}+u_{j, i}\right)
$$

The heat conduction

$$
\kappa I^{\xi-1} \varphi_{i i}=\left(\frac{\partial}{\partial t}-\tau_{o} \frac{\partial^{2}}{\partial t^{2}}\right)\left(\rho C_{E} \theta+\gamma T_{o} e\right)
$$


where

$$
I^{\xi} f(t)= \begin{cases}\frac{1}{\Gamma(\xi)} \int_{0}^{t}(t-\tau)^{\xi-1} f(\tau) \mathrm{d} \tau, & 0<\xi \leq 2, \\ f(t), & \xi=0\end{cases}
$$

The thermodynamical temperature $\theta$ relates with the conductive temperature $\varphi$ by the relation

$$
\varphi-\theta=a \varphi_{i i},
$$

in which $a>0$ is the two-temperature parameter.

In the above equations, a comma followed by a suffix denotes material derivatives and a superposed dot denotes the derivatives with respect to time.

\section{One Dimension Formulation}

Consider a semi-infinite piezoelectric rod occupying the region $x \geq 0$. At the near end a uniform flow of heat is supplied to the rod during a finite period of time. All the state functions field will depend only on the dimension $x$ and the time $t$. We assume the following form for the displacement component:

$$
u_{x}=u(x, t), u_{y}=u_{z}=0
$$

We consider the following forms of the linearized basic equations in one-dimensional formulation:

$$
\begin{gathered}
(\lambda+2 \mu) \frac{\partial^{2} u}{\partial x^{2}}-\gamma \frac{\partial \theta}{\partial x}=\rho \frac{\partial^{2} u}{\partial t^{2}} \\
\sigma=(\lambda+2 \mu) \frac{\partial u}{\partial x}-\gamma \theta-h D \\
\kappa I^{\xi-1} \frac{\partial^{2} \varphi}{\partial x^{2}}=\left(\frac{\partial}{\partial t}-\tau_{o} \frac{\partial^{2}}{\partial t^{2}}\right)\left(\rho C_{E} \theta+\gamma T_{o} e\right) \\
\theta=\varphi-a \frac{\partial^{2} \varphi}{\partial x^{2}} \\
e=\frac{\partial u(x, t)}{\partial x} \\
\frac{\partial D}{\partial x}=0 \\
E=-\frac{\partial v}{\partial x},
\end{gathered}
$$

where $\gamma=\alpha_{t}(3 \lambda+2 \mu), \quad \alpha_{t}$ is the coefficient of the linear thermal expansion, $\kappa$ is the coefficient of thermal conductivity and $x$ is the coordinate taken along the rod.

It is convenient now to introduce the following dimensionless variables:

$$
\begin{aligned}
& u^{\prime}=c_{o} \eta u, t^{\prime}=c_{o}^{2} \eta t, \sigma^{\prime}=\frac{\sigma}{(\lambda+2 \mu)}, \theta^{\prime}=\frac{T-T_{o}}{T_{o}}, \\
& t_{o}^{\prime}=c_{o}^{2} \eta t_{o}, x^{\prime}=c_{o} \eta x, \tau^{\prime}=c_{o}^{2} \eta \tau, \varphi^{\prime}=\frac{\varphi-T_{o}}{T_{o}} \\
& q^{\prime}=\frac{q}{k c_{o} \eta T_{o}}, D^{\prime}=\frac{h}{\lambda+2 \mu} D, \eta=\frac{\rho C_{E}}{k}, c_{o}^{2}=\frac{\lambda+2 \mu}{\rho}
\end{aligned}
$$

From Gauss's law, since there is no free charge inside the piezoelectric rod we have

$$
\frac{\partial D}{\partial x}=0
$$

which gives

$$
D=\text { const }
$$

Substituting from Equation (26) into Equations (19)(25) and dropping the primes for convenience, we obtain the following set of non-dimensional equations Youssef [29] and Youssef and Bassiouny [30] follows:

$$
\begin{gathered}
\frac{\partial^{2} e}{\partial x^{2}}-\alpha \frac{\partial^{2} \theta}{\partial x^{2}}=\frac{\partial^{2} e}{\partial t^{2}} \\
\sigma=e-\alpha \theta-D \\
I^{\xi-1} \frac{\partial^{2} \varphi}{\partial x^{2}}=\left(\frac{\partial}{\partial t}-\tau_{o} \frac{\partial^{2}}{\partial t^{2}}\right)(\theta+\varepsilon e)
\end{gathered}
$$

and the following relation between the conductive temperature and the thermodynamical one:

$$
\theta=\varphi-\omega \frac{\partial^{2} \varphi}{\partial x^{2}}
$$

where

$$
\theta=\varphi-\omega \frac{\partial^{2} \varphi}{\partial x^{2}}, \varepsilon=\frac{\gamma}{\rho C_{E}}, \text { and } \omega=a c_{o}^{2} \eta^{2}
$$

The boundary conditions are:

$$
\begin{gathered}
\varphi(0, t)=\varphi_{o} \frac{\partial^{\xi}\left(1-e^{-\Omega t}\right)}{\partial t^{\xi}} \\
\varphi(\infty, t)=0, e(0, t)=e(\infty, t)=0,0<t<\infty \\
\sigma(\infty, t)=0, \sigma(0, t)=0,0<t<\infty
\end{gathered}
$$

where $0 \leq \xi<2$, while the initial conditions are assumed to be:

$$
\varphi(x, 0)=0, e(x, 0)=0, \sigma(x, 0)=0,0 \leq x \leq \infty
$$

Applying the Laplace transform defined by:

$$
L\{f(t)\}=\overline{f(s)}=\int_{0}^{\infty} e^{-s t} f(t) \mathrm{d} t
$$

to both sides of Equations (29)-(32), we obtain: 


$$
\begin{gathered}
\frac{\mathrm{d}^{2} \bar{e}}{\mathrm{~d} x^{2}}-\alpha \frac{\mathrm{d}^{2} \bar{\theta}}{\mathrm{d} x^{2}}=s^{2} \bar{e} \\
\bar{\sigma}=\bar{e}-\alpha \bar{\theta}-\frac{D}{s} \\
\frac{1}{s^{\xi-1}} \frac{\mathrm{d}^{2} \bar{\varphi}}{\mathrm{d} x^{2}}=\left(s+\tau_{o} s^{2}\right) \bar{\theta}+\varepsilon\left(s+\tau_{o} s^{2}\right) \bar{e} \\
\bar{\theta}=\bar{\varphi}-\omega \frac{\mathrm{d}^{2} \bar{\varphi}}{\mathrm{d} x^{2}} .
\end{gathered}
$$

Using Equations (29) and (30) with the definitions (23) and (37) we can obtain

$$
\bar{u}=\bar{s}^{2} \frac{\mathrm{d} \bar{\sigma}}{\mathrm{d} x}
$$

where $s$ denotes the complex argument related to the Laplace transform.

The transformed boundary conditions take the forms

$$
\overline{\varphi(0, s)}=\varphi_{o} \frac{\overline{\partial^{\xi}\left(1-e^{\Omega t}\right)}}{\partial^{\xi} t}=F(s)
$$

where

$$
F(s)=\varphi_{o}\left(s^{-1-\xi}-(s+\Omega)^{-1-\xi}\right)
$$

while the Equations (35) become

$$
\begin{aligned}
& \bar{\varphi}(\infty, s)=0, \bar{e}(0, s)=\bar{e}(\infty, s)=0, \\
& \bar{\sigma}(\infty, s)=0, \quad \bar{\sigma}(0, s)=0,
\end{aligned}
$$

and the corresponding transformed initial conditions of the Equations (36) assume the form:

$$
\bar{\varphi}(x, 0)=\bar{e}(x, 0)=\bar{\sigma}(x, 0)=0, \quad 0 \leq x \leq \infty
$$

Eliminating $\bar{\theta}$ between Equations (40) and (41), we get:

$$
D^{2} \bar{\varphi}=L \bar{\varphi}+L \varepsilon \bar{e}
$$

where

$$
L=L(s)=\frac{s+\tau_{o} s^{2}}{s^{1-\xi}+\omega\left(s+\tau_{o} s^{2}\right)} \text { and } D^{2}=\frac{\mathrm{d}^{2}}{\mathrm{~d} x^{2}}
$$

Substituting from Equation (47) into Equation (41) we obtain

$$
\bar{\theta}=(1-\omega L) \bar{\varphi}-\omega L \varepsilon \bar{e}
$$

Using Equation (47) we can easily eliminate $\bar{\theta}$ between Equation (38) and (48) to obtain

$$
D^{2} \bar{e}=M \bar{\varphi}+N \bar{e}
$$

where

$$
\begin{aligned}
& M=M(s)=\frac{\alpha L(1-\omega L)}{1+\omega \alpha \varepsilon L} \\
& \text { and } N=N(s)=\frac{s^{2}+\alpha \varepsilon L(1-\omega L)}{1+\omega \alpha \varepsilon L}
\end{aligned}
$$

Solving Equations (47) and (49) together we get the following fourth order equation

where

$$
\left[k^{4}-a k^{2}+b\right] \bar{\varphi}=0
$$

$$
\begin{gathered}
a=\frac{s^{2+\xi}\left(1+s \tau_{o}\right)}{1+s^{\xi}(1+\alpha \varepsilon)\left(1+s \tau_{o}\right) \omega} \\
b=\frac{s^{2}+s^{\xi}\left(1+s \tau_{o}\right)\left(1+\alpha \varepsilon+\omega s^{2}\right)}{1+s^{\xi}(1+\alpha \varepsilon)\left(1+s \tau_{o}\right) \omega}
\end{gathered}
$$

It is worth mentioning here that the roots of equation (51) are functions of $s$ and assume the forms:

$$
k_{1}= \pm \frac{\sqrt{a+\sqrt{a^{2}-4 b}}}{\sqrt{2}}, k_{2}= \pm \frac{\sqrt{a-\sqrt{a^{2}-4 b}}}{\sqrt{2}}
$$

Thus the solutions of the Equations (47) and (49) satisfying the boundary conditions at infinity are:

$$
\begin{gathered}
\bar{\varphi}=A_{1} e^{-x k_{1}}+A_{2} e^{-x k_{2}} \\
\bar{e}=B_{1} e^{-x k_{1}}+B_{2} e^{-x k_{2}}
\end{gathered}
$$

where $A_{1}, A_{2}, B_{1}$ and $B_{2}$ are coefficients depending on $s$ to be determined using the boundary conditions (43) and (45).

$$
\begin{aligned}
& A_{1}=\frac{-F(s)}{N(s)\left(k_{1}^{2}-k_{2}^{2}\right)}\left[N k_{2}^{2}-s^{-\xi}\left(1+s \tau_{o}\right)\right] \\
& A_{2}=\frac{F(s)}{N(s)\left(k_{1}^{2}-k_{2}^{2}\right)}\left[N k_{1}^{2}-s^{-\xi}\left(1+s \tau_{o}\right)\right]
\end{aligned}
$$

While the constants $B_{1}, B_{2}$ are related to the constants $A_{1}, A_{2}$ according to the following relations:

$$
B_{i}=\frac{A_{i}}{\varepsilon\left(1+s \tau_{o}\right)}\left[k_{i}^{2}\left(s^{-\xi}+\left(1+s \tau_{o}\right) \omega\right)-\left(1+s \tau_{o}\right)\right], i=1,2
$$

Substituting from Equations (57) and (58) into the Equations (55) and (56) the heat conduction and the strain field in the Laplace domain take the forms:

$$
\begin{gathered}
\bar{\varphi}(x, s)=\frac{F(s)}{N(s)\left(k_{1}^{2}-k_{2}^{2}\right)} \\
\left\{\left(s^{-\xi}\left(1+s \tau_{o}\right)-N k_{2}^{2}\right) e^{-x k_{1}}+\left(s^{-\xi}\left(1+s \tau_{o}\right)+N k_{1}^{2}\right) e^{-x k_{2}}\right\} \\
\bar{e}(x, s)=\frac{1}{\varepsilon\left(1+s \tau_{o}\right)} \\
{\left[A_{1}\left(k_{1}^{2}\left(s^{-\xi}+\left(1+s \tau_{o}\right) \omega\right)-\left(1+s \tau_{o}\right)\right) e^{-x k_{1}}\right.} \\
\left.+A_{2}\left(k_{2}^{2}\left(s^{-\xi}+\left(1+s \tau_{o}\right) \omega\right)-\left(1+s \tau_{o}\right)\right) e^{-x k_{2}}\right]
\end{gathered}
$$

Using the expressions of $\bar{\varphi}$ and of $\bar{e}$ from Equations (60) and (61) to find the thermodynamical function 
$\bar{\theta}$ and the stress in the Laplace transformed domain, thus Equations (39) and (48) become:

$$
\begin{gathered}
\bar{\sigma}(x, s)=\sigma_{1} e^{-x k_{1}}+\sigma_{2} e^{-x k_{2}}-\frac{D}{s} \\
\bar{\theta}(x, s)=\theta_{1} e^{-x k_{1}}+\theta_{2} e^{-x k_{2}} \\
\bar{u}(x, s)=u_{1} e^{-x k_{1}}+u_{2} e^{-x k_{2}}
\end{gathered}
$$

where

$$
\begin{gathered}
\sigma_{i}=\frac{A_{i}}{1+s \tau_{o}}\left[k_{i}^{2}\left(s^{-\xi}+\left(1+s \tau_{o}\right)(1+\alpha \varepsilon) \omega\right)-(1+\alpha \varepsilon)\right] \\
\theta_{i}=A_{i}\left(1-k_{i}^{2} \omega\right) \\
u_{i}=-s^{-2} k_{i} \sigma_{i}
\end{gathered}
$$

Equations (60)-(64) are the complete solutions of the $\bar{\varphi}, \bar{e}, \bar{\theta}, \bar{\sigma}$ and $\bar{u}$, respectively, in the Laplace transformed domain.

In order to invert the Laplace transform, we adopt a numerical inversion method based on a Fourier series expansion Honig [31]. Using this method, the inverse $f(t)$ of the Laplace transform $\bar{f}(s)$ is approximated by

$$
\begin{aligned}
& f(t)=\frac{e^{c t}}{t_{1}}\left[\frac{1}{2} \bar{f}(c)+\operatorname{Re} \sum_{k=1}^{N} \bar{f}\left(c+\frac{i k \pi}{t_{1}}\right) \exp \left(\frac{i k \pi t}{t_{1}}\right)\right], \\
& 0<t_{1}<2 t
\end{aligned}
$$

where $N$ is a sufficiently large integer representing the number of terms in the truncated Fourier series, chosen such that

$$
\exp (c t) \operatorname{Re}\left[\bar{f}\left(c+\frac{i N \pi}{t_{1}}\right) \exp \left(\frac{i N \pi t}{t_{1}}\right)\right] \leq \varepsilon_{1}
$$

where $\varepsilon_{1}$ is a prescribed small positive number that corresponds to the degree of accuracy required and Re is the real part. The parameter $\mathrm{c}$ is a positive free parameter that must be greater than the real part of all the singularities of $\bar{f}(s)$. The optimal choice of $\mathrm{c}$ was obtained according to the criteria described in [31].

\section{Numerical Results and Discussion}

To investigate the role of various physical parameters involved in the current problem, we have investigated the role of varying the angular frequency of thermal vibration $\Omega$ on different system parameters, where it is observed that increasing $\Omega$ increases the heat conduction, as depicted in Figure 1(a). Such behavior is in accordance with the fact that increasing the thermal vibrations will increase the kinetic energy of the ceramic slab molecules and results in increasing the amount of heat transferred by conduction mechanism. Variation of the
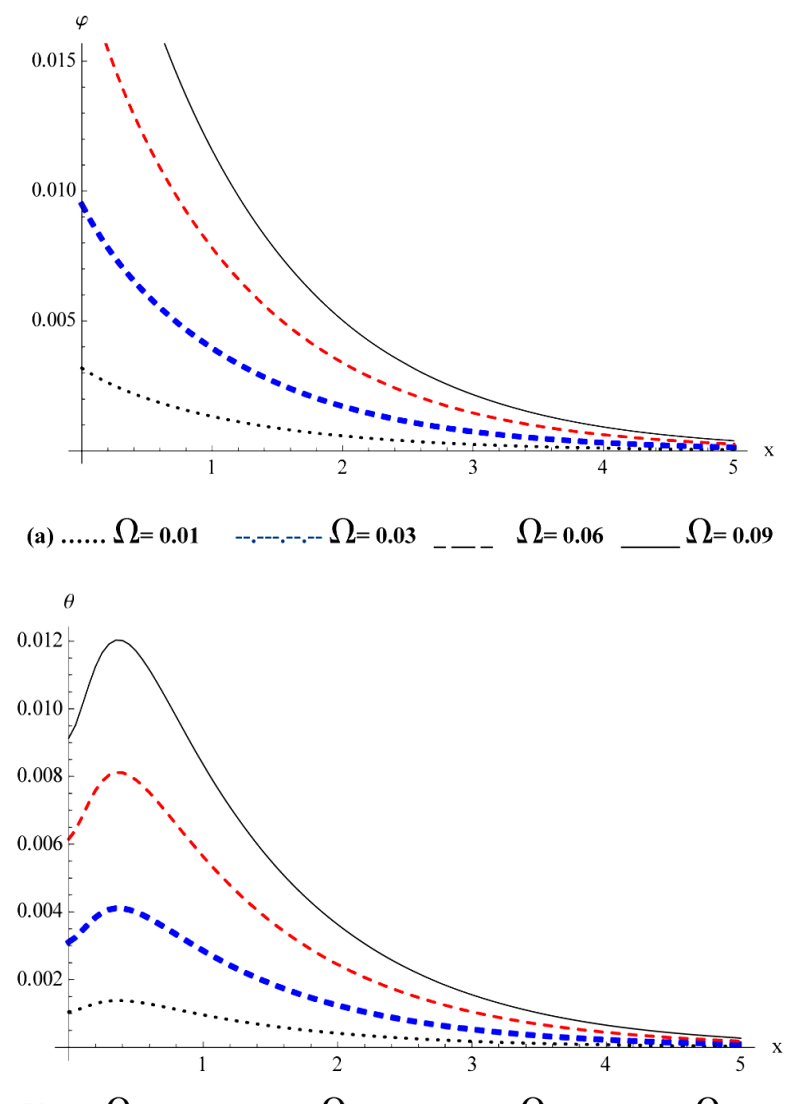

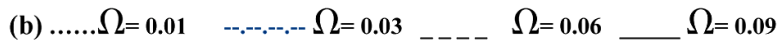

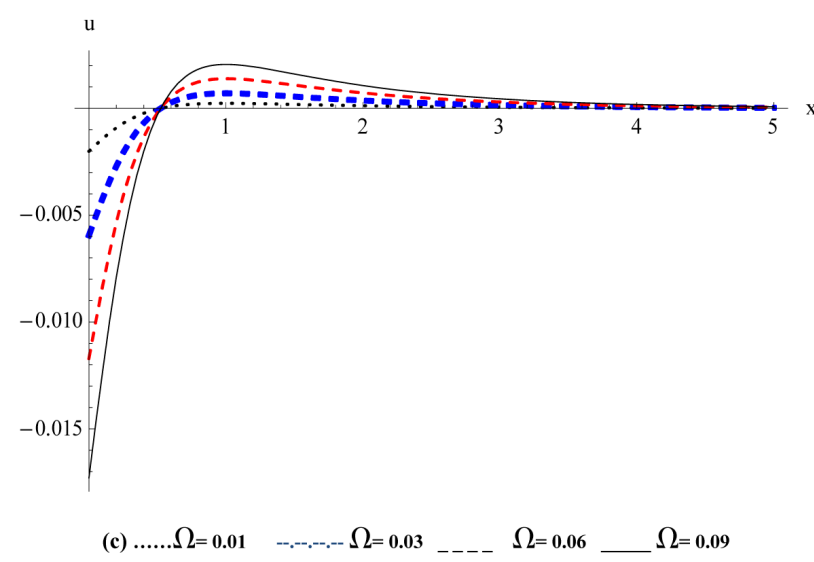

Figure 1. Variation of (a) Heat conduction $\varphi$ (b) Thermodynamical temperature and (c) Displacement against $x$ for various values of angular frequency of thermal vibration $\Omega$ at $t=0.3, \omega=0.4, \tau_{0}=0.3, \xi=0.75$.

thermodynamical temperature for different $\Omega$, shows a peculiar behavior, as illustrated in Figure 1(b) and reflected through the variation of the displacement distribution shown in Figure 1(c). It is found that increasing the thermal vibrations will increase the amplitude of the thermodynamic temperature. The effect of increasing the thermal vibrations frequency on the stress and strain shows the same qualitative behavior, as illustrated in 
Figure 2. Both of the stress and strain decreases initially by increasing $x$, but at a certain critical point further increase in $x$ increases the stress and strain. It is observed in Figure 2 that the thermal vibration increases the stress and strain in a symmetrical way with respect to a critical

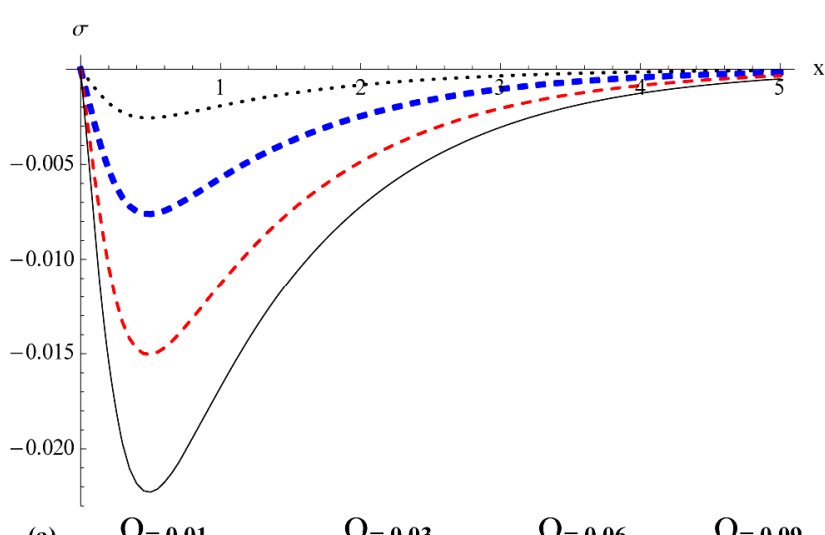

point.

The effect of increasing time is shown to increase the heat conduction as well as the thermodynamic temperature, as reflected in Figures 3(a) and (b), respectively. In fact it shows the same qualitative behavior of increasing

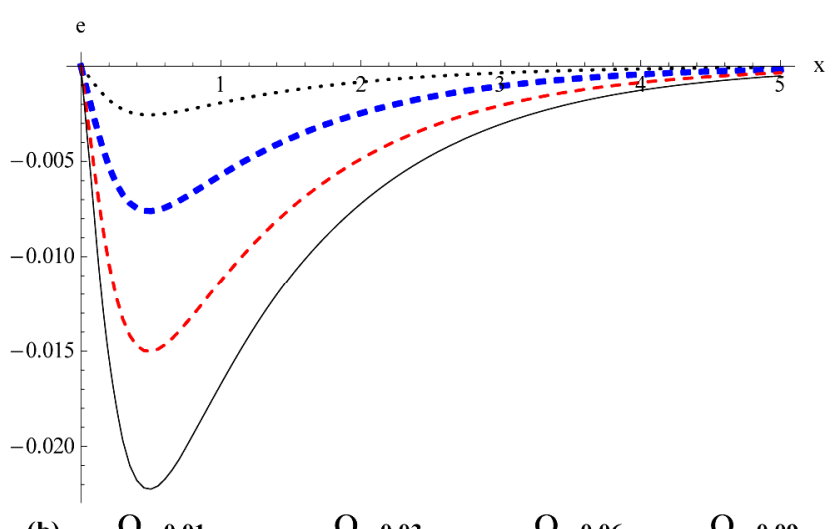

Figure 2. The role of varying angular frequency $\Omega$ on the (a) Stress and (b) Strain at $t=0.3, \omega=0.4, \tau_{0}=0.3, \xi=0.75$.
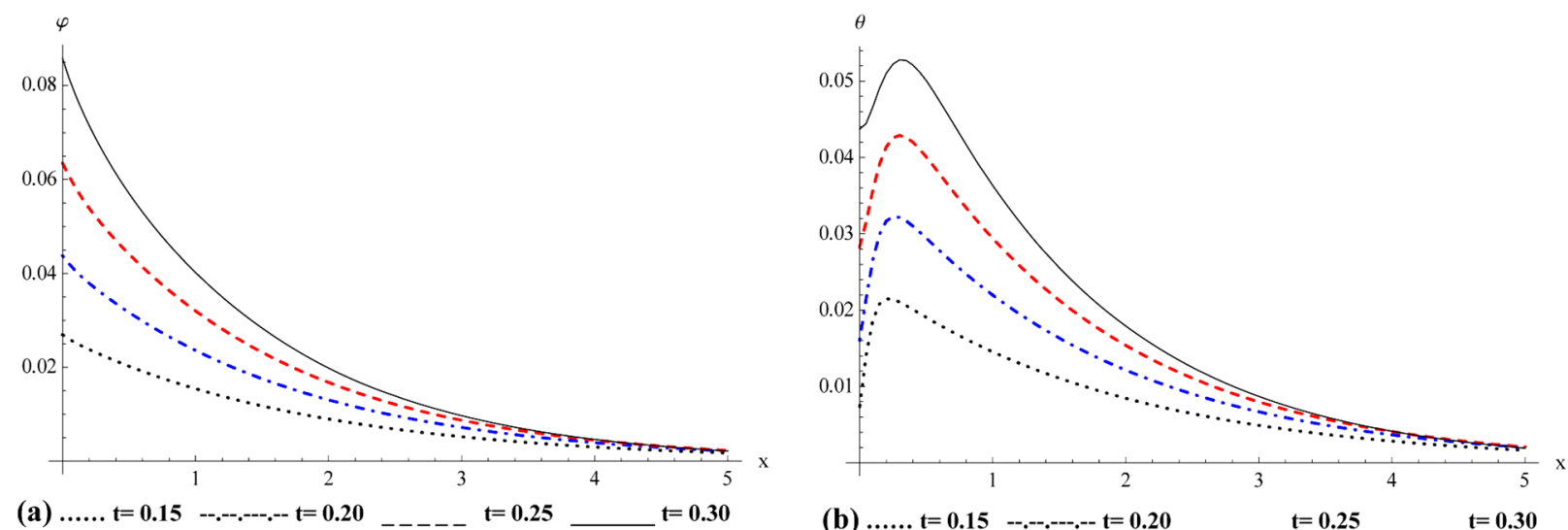

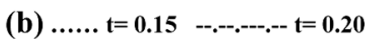
$\mathbf{t}=\mathbf{0 . 2 5}$ $\mathbf{t}=\mathbf{0 . 3 0}$

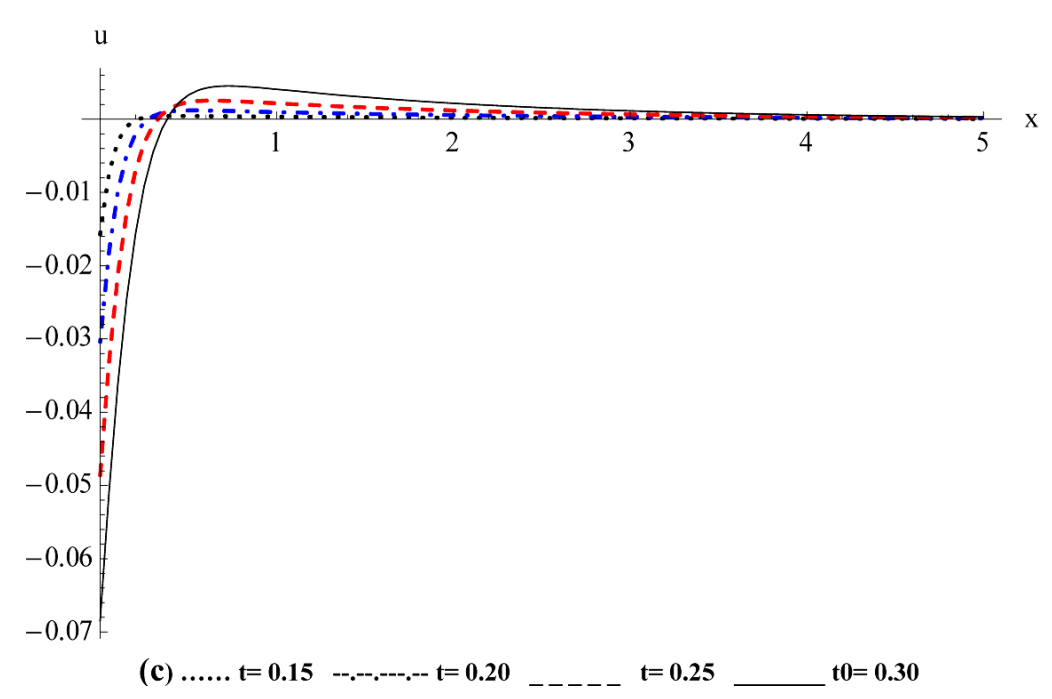

Figure 3. Variation of (a) Heat conduction $\varphi$ (b) Thermodynamical temperature and (c) Displacement against $x$ for different value of time at $\omega=0.2, \tau_{0}=0.3, \xi=0.75, \Omega=0.1$. 
the frequency of the thermal vibrations on the heat conduction, illustrated in Figure 1(a). Such behavior can be explained on the basis that increasing the time of heating the slab will increase the amount of energy delivered to the slab. The amount of energy delivered to the ceramic slab increases the entropy of the thermodynamic temperature. The minimum points of the stress and strain curves are shown to be an increasing function in $x$ as time increases. Far from the near end of the slab the effect of time damped as $x$ increases and the stress and strain will be an increasing function in $x$, as illustrated in Figure 4. The amount of energy delivered to the ceramic slab is a factor of heating time, which is the key answer to such behavior. An inverse proportion is noticed between the value of the fractional order (i.e., the measure of the system memory) and the heat conduction at the near end of the slab, whereas a slight change in the distribution curves is noticed for large values of $x$, as depicted in Figure 5(a). The thermodynamic temperature is a decreasing function in the fractional order as shown in

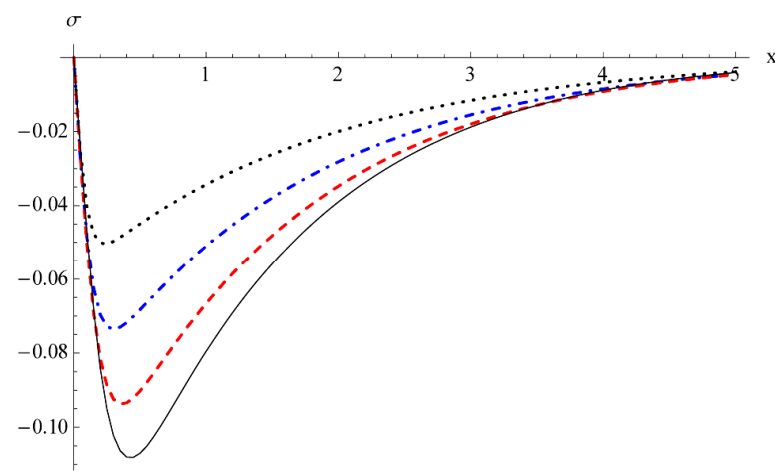

(a)

$\mathrm{t}=0.15-\cdots,-. .---\mathrm{t}=0.20$

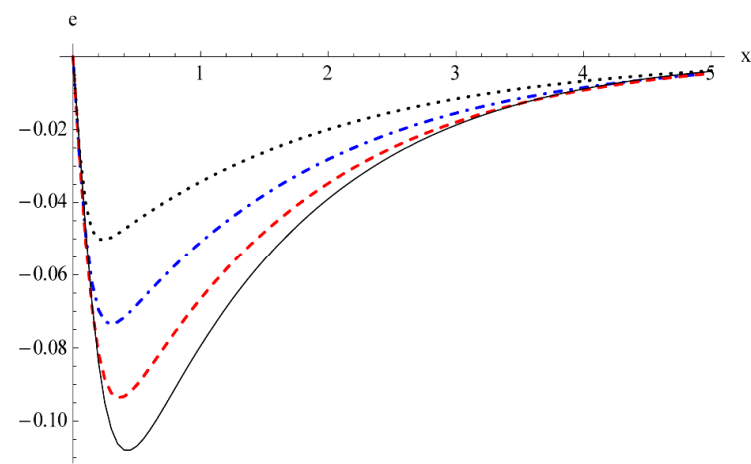

Figure 4. The role of varying time on the (a) Stress and (b) Strain at $\omega=0.2, \tau_{0}=0.3, \xi=0.75, \Omega=0.1$.
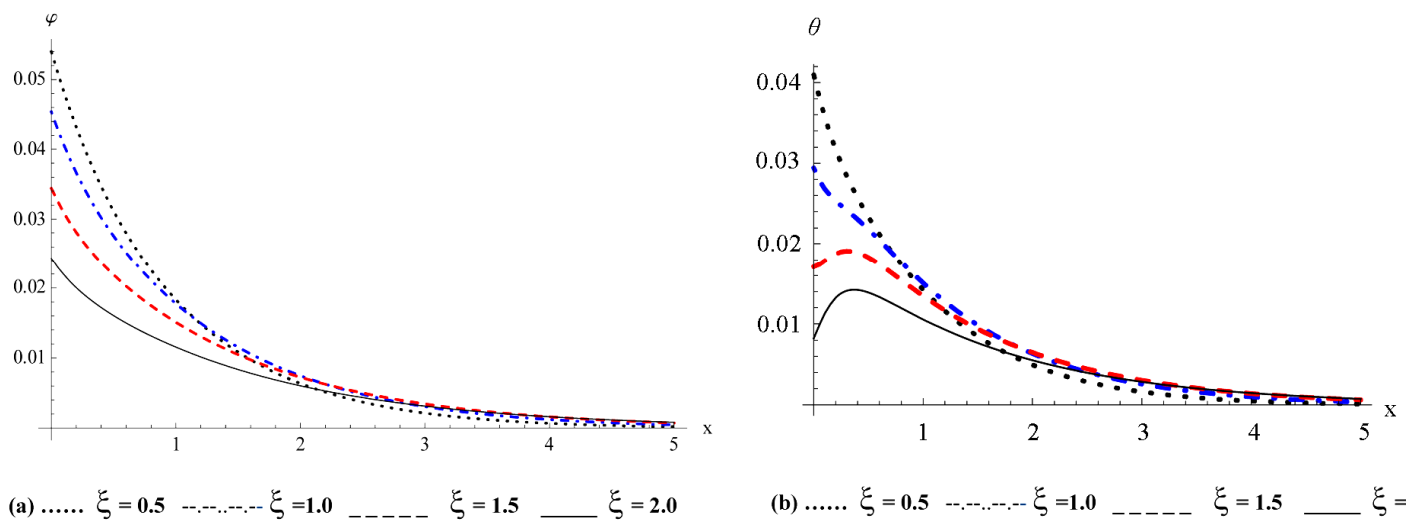

(b) $\ldots \ldots . . . \xi=0.5-\ldots-\ldots-.--\xi=1.0$ $\xi=1.5-\xi=2.0$

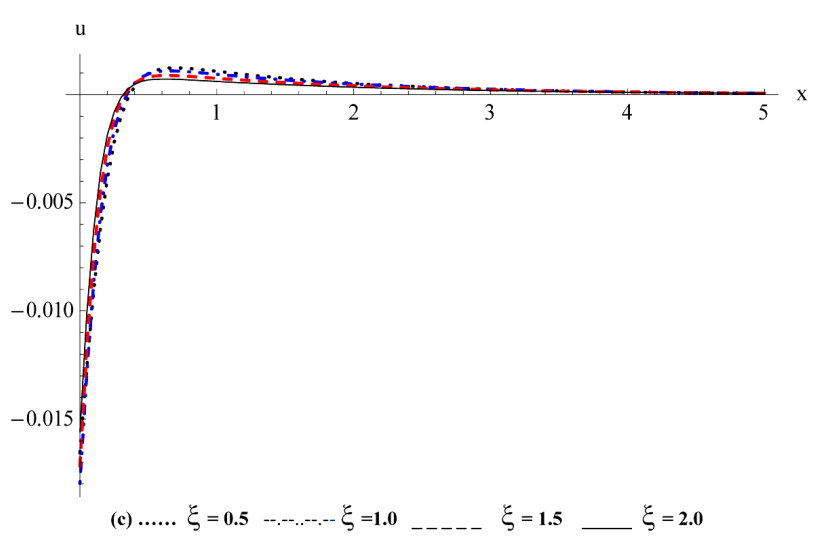

Figure 5. Variation of (a) Heat conduction $\varphi$, (b) Thermodynamical temperature and (c) Displacement against $x$ for different fractional order parameter at $t=0.4, \omega=0.2, \tau_{0}=0.3, \Omega=0.1$. 
Figure 5(b). It is apparent that increasing the system fractional order leads to reverse the effect of heating as in Figure 5. The qualitative behavior of the displacement distribution is shown to resemble the same behavior as in the case of the effects of thermal angular vibration and time but here; the fractional has a slight effect on the

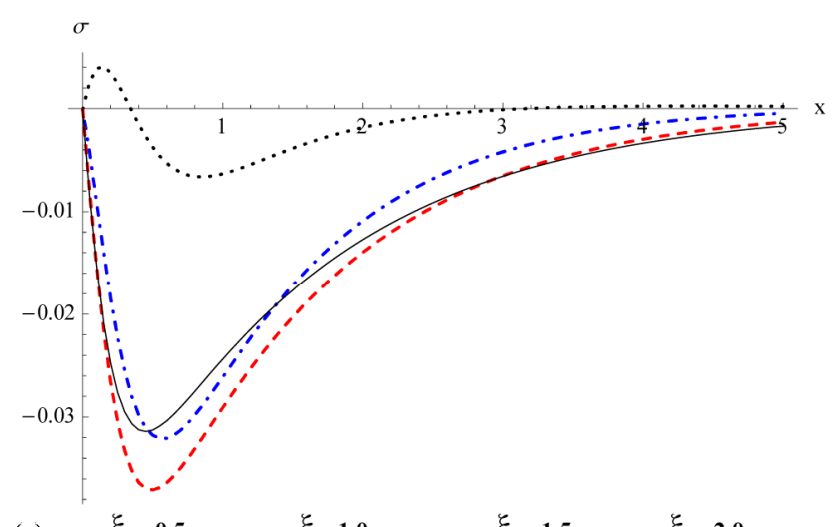

displacement distribution. The amount of energy delivered to the ceramic slab is affected by the weak conductivity imposed on the material by the system memory retained through the fractional parameter. Such behavior is confirmed through the role of the fractional parameter on the stress and strain curves as displayed in Figure 6.

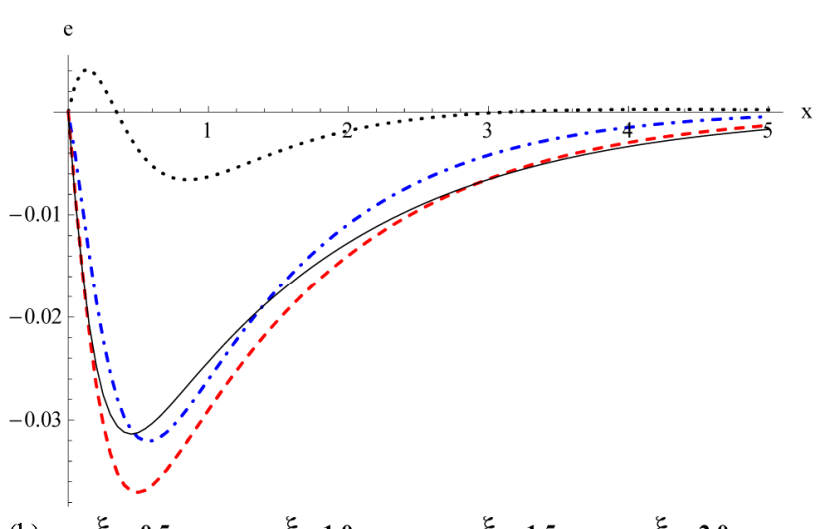

(b)..... $\xi=0.5$-...-..-.-- $\xi=1.0 \quad \ldots \quad \xi=1.5 \_\xi=2.0$

Figure 6. The role of varying fractional order parameter on the (a) Stress and (b) Strain at $t=0.4, \omega=0.2, \tau_{0}=0.3, \Omega=0.1$.
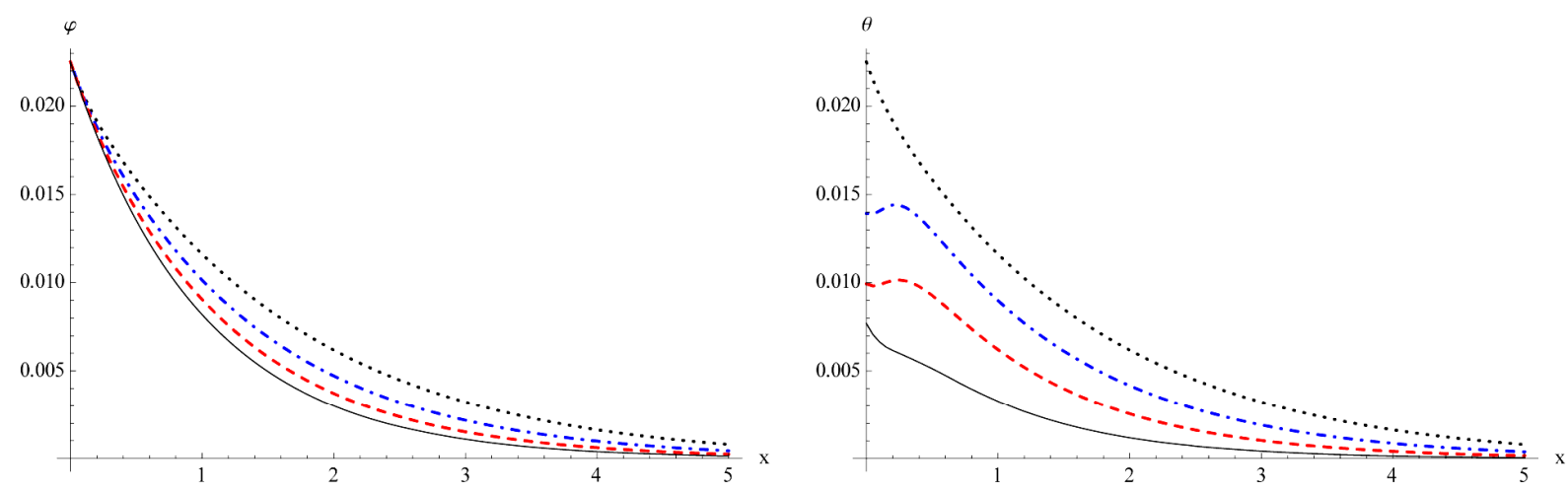

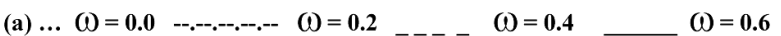

(b) $\ldots \omega=0.0$

$\omega=0.2$

$\omega=0.4$

$\omega=0.6$

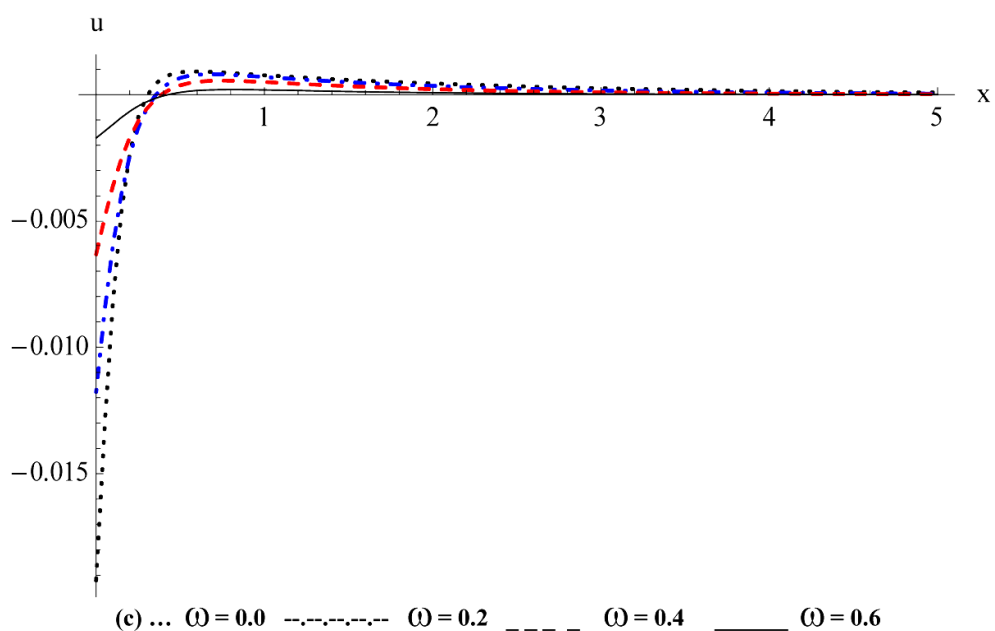

Figure 7. Variation of (a) Heat conduction $\varphi$; (b) Thermodynamic temperature $\theta$; (c) Displacement $U$ against $x$ for different value of the two-temperature parameter at $t=0.25, \xi=0.75, \tau_{0}=0.3, \Omega=0.1$. 

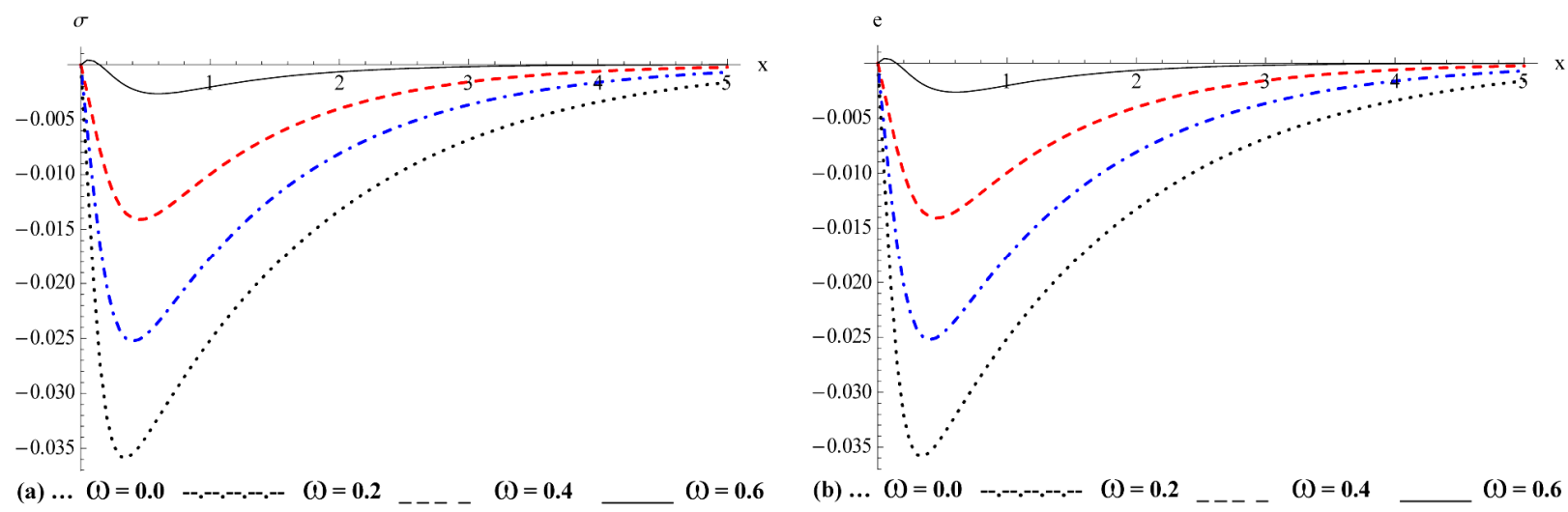

Figure 8. The role of varying the two-temperature parameter $\omega$ on the (a) Stress and (b) Strain at $t=0.25$, $\xi=0.75, \tau_{0}=0.3, \Omega=0.1$.

The two temperature parameter $\omega$, which depends on two distinct temperatures, the conductive temperature and the thermodynamic temperature where the difference between these two temperatures is proportional to the heat supply, is found to increase the heat conduction as shown in Figure 7(a). The thermodynamic temperature increases by increasing $\omega$ as illustrated in Figure 7(b). The displacement is found to have a critical behavior at $x \approx 0.4$, as depicted in Figure 7(c) where the displacement distribution curves for all values of $\omega$ pass by this critical point and the distribution becomes positive after it. The strain distribution confirms similar qualitative behavior as the stress distribution as illustrated in Figure 8. Both distributions of the stress and strain are decreased by increasing $\omega$ for $\omega \leq 0.4$, as shown in Figure 8 . However, for $\omega>0.4$ the stress and strain distributions remain decreasing functions in the two temperature parameter $\omega$.

\section{REFERENCES}

[1] P. J. Chen and M. E. Gurtin, "On a Theory of Heat Conduction Involving Two Temperatures," Zeitschrift für Angewandte Mathematik und Physik ZAMP, Vol. 19, No. 4, 1968, pp. 614-627. http://dx.doi.org/10.1007/BF01594969

[2] P. J. Chen, M. E. Gurtin and W. O. Williams, "A Note on Non-Simple Heat Conduction," Zeitschrift für Angewandte Mathematik und Physik ZAMP, Vol. 19, No. 6, 1968, pp. 969-970. http://dx.doi.org/10.1007/BF01602278

[3] P. J. Chen, M. E. Gurtin and W. O. Williams, "On the Thermodynamics of Non-Simple Elastic Materials with Two Temperatures," Zeitschrift für Angewandte Mathematik und Physik ZAMP, Vol. 20, No. 1, 1969, pp. 107112. http://dx.doi.org/10.1007/BF01591120

[4] B. A. Boley and I. S. Tolins, "Transient Coupled Thermoelastic Boundary Value Problems in the Half-Space," Journal of Applied Mechanics, Vol. 29, No. 4, 1962, pp.
637-646. http://dx.doi.org/10.1115/1.3640647

[5] W. E. Warren and P. J. Chen, "Wave Propagation in the Two-Temperature Theory of Thermoelasticity," Acta Mechanica, Vol. 16, No. 1-2, 1973, pp. 21-33. http://dx.doi.org/10.1007/BF01177123

[6] H. M. Youssef, "Theory of Two-Temperature Generalized Thermoelasticity," IMA Journal of Applied Mathematics, Vol. 71, No. 3, 2006, pp. 383-390. http://dx.doi.org/10.1093/imamat/hxh101

[7] M. Caputo, "Linear Models of Dissipation Whose Q Is almost Frequently Independent II," Geophysical Journal International, Vol. 13, No. 5, 1967, pp. 529-539. http://dx.doi.org/10.1111/j.1365-246X.1967.tb02303.x

[8] F. Mainardi, "Fractional Calculus: Some Basic Problems in Continuum and Statistical Mechanics," In: A. Carpinteri and F. Mainardi, Eds., Fractals and Fractional Calculus in Continuum Mechanics, Springer, New York, 1997, pp. 291-348.

[9] I. Podlubny, "Fractional Differential Equations," Academic Press, New York, 1999.

[10] R. Hilfer, "Application of Fraction Calculus in Physics," World Scientific, Singapore, 2000.

[11] M. Caputo and F. Mainardi, "Linear Model of Dissipation in Inelastic Solids," Rivis Ta El Nuovo Cimento, Vol. 1, No. 2, 1971, pp. 161-198. http://dx.doi.org/10.1007/BF02820620

[12] M. Caputo, "Vibrations of an Infinite Viscoelastic Layer with a Dissipative Memory," The Journal of the Acoustical Society of America, Vol. 56, 1974, pp. 897-904. http://dx.doi.org/10.1121/1.1903344

[13] R. L. Bagley and P. J. Torvik, "A Theoretical Basis for the Application of Fractional Calculus to Viscoelasticity," Journal of Rheology, Vol. 27, 1983, pp. 201-210. http://dx.doi.org/10.1122/1.549724

[14] R. C. Koeller, "Applications of Fractional Calculus to the Theory of Viscoelasticity," Journal of Applied Mechanics, Vol. 51, No. 2, 1984, pp. 299-307. http://dx.doi.org/10.1115/1.3167616

[15] Yu. A. Rossikhin and M. V. Shitikova, "Applications of Fractional Calculus to Dynamic Problems of Linear and 
Nonlinear Heredity Mechanics of Solids," Applied Mechanics Reviews, Vol. 50, No. 1, 1997, pp. 15-67. http://dx.doi.org/10.1115/1.3101682

[16] H. H. Sherief, A. El-Said and A. Abd El-Latief, "Fractional Order Theory of Thermoelasticity," International Journal of Solids and Structures, Vol. 47, No. 2, 2010, pp. 269-275. http://dx.doi.org/10.1016/j.ijsolstr.2009.09.034

[17] M. A. Ezzat and A. S. El-Karamany, "On the Fractional Thermo-Elasticity," Mathematics and Mechanics of Solid, 2011.

[18] I. Podlubny, "Fractional Differential Equations," Academic Press, New York, 1999.

[19] R. Kimmich, "Strange Kinetics, Porous Media, and NMR," Journal of Chemical Physics, Vol. 284, 2002, pp. 243-285.

[20] Y. Fujita, "Integrodifferential Equation which Interpolates the Heat Equation and Wave Equation (II)," Osaka Journal of Mathematics, Vol. 27, 1990, pp. 797-804.

[21] Y. Fujita, "Integrodifferential Equation which Interpolates the Heat Equation and Wave Equation (I)," Osaka Journal of Mathematics, Vol. 27, 1990, pp. 309-321.

[22] Y. Z. Povstenko, "Fractional Heat Conductive and Associated Thermal Stress," Journal of Thermal Stresses, Vol. 28, No. 1, 2004, pp. 83-102. http://dx.doi.org/10.1080/014957390523741

[23] Y. Z. Povstenko, "Theories of Thermal Stresses Based on Space-Time-Fractional Telegraph Equations," Computers and Mathematics with Applications, Vol. 64, No. 10, 2012, pp. 3321-3328.

http://dx.doi.org/10.1016/j.camwa.2012.01.066

[24] C. Cattaneo, "Sur une Forme de I'equation de la Chaleur Eliminant le Paradoxe d'une Propagation Instantanee'," Comptes Rendus de l'Académie des Sciences, Vol. 247, 1958, pp. 431-433.
[25] M. A. Ezzat and A. S. El-Karamany, "Discontinuities in Generalized Thermoviscoelasticity under Four Theories," Journal of Thermal Stresses, Vol. 27, No. 12, 2004, pp. 1187-1212. http://dx.doi.org/10.1080/014957390523598

[26] M. A. Ezzat and A. S. El-Karamany, "Fractional Order Theory of a Prefect Conducting Thermoelastic Medium," Canadian Journal of Physics, Vol. 89, No. 3, 2011, pp. 311-318. http://dx.doi.org/10.1139/P11-022

[27] M. A. Ezzat and A. S. El-Karamany, "Theory of Fractional Order in Electro-Thermoelasticity," European Journal of Mechanics A/Solids, Vol. 30, No. 4, 2011, pp. 491500. http://dx.doi.org/10.1016/j.euromechsol.2011.02.004

[28] A. S. El-Karamany and M. A. Ezzat, "Convolutional Variational Principle, Reciprocal and Uniqueness Theorems in Linear Fractional Two-Temperature Thermoelasticity," Journal of Thermal Stresses, Vol. 34, No. 3, 2011, pp. 264-284.

http://dx.doi.org/10.1080/01495739.2010.545741

[29] H. Youssef, "Theory of Fractional Order Generalized Thermoelasticity," Journal of Heat Transfer, Vol. 132, No. 6, 2010, pp. 1-7. http://dx.doi.org/10.1115/1.4000705

[30] H. M. Youssef and E. Bassiouny, "Two-Temperature Generalized Thermopiezoelasticity for One Dimensional Problems-State Space Approach," Computational Methods in Science and Technology, Vol. 14, No. 1, 2008, pp. 5564.

[31] G. Honig and U. Hirdes, "A Method of the Numerical Inversion of Laplace Transform," Journal of Computational and Applied Mathematics, Vol. 10, No. 1, 1984, pp. 113-132.

http://dx.doi.org/10.1016/0377-0427(84)90075-X 


\section{Nomenclatures}

$A_{i j}$ : The components of relaxation time.

$a$ : The two-temperature parameter.

$C_{E}$ : Specific heat at constant strain.

$c_{i j k l}:$ The elastic constants.

$c_{o}=\sqrt{\frac{\lambda+2 \mu}{\rho}}:$ Longitudinal wave speed.

$D_{i}$ : The components of electric displacement.

$d_{i}$ : The pyroelectric constants.

$E_{i}$ : The components of electric field vector.

$e_{i j k l}$ : The components of strain tensor. conductivity.

$h_{i j k}$ : The piezoelectric coefficients.

$k_{i j}$ : The components of thermal.

$q_{i}$ : The components of the heat flux vector.

$T:$ Absolute temperature.

$T_{o}$ : Reference temperature.

$t$ : Time.

$u_{i}$ : Components of displacement vector.

$v_{i}$ : The electric potential function.

$\alpha=\frac{\gamma T_{o}}{\lambda+2 \mu}:$ Dimensionless thermoelastic coupling constant.
$\alpha_{T}$ : Coefficient of linear thermal expansion.

$\tau_{i k}$ : The components of dielectric tensor.

$\beta_{i j}$ : The thermal modulus.

$\gamma=(3 \lambda+2 \mu) \alpha_{T}$.

$\Omega$ : The angular frequency of thermal vibration.

$\delta_{i j}$ : Kronecker delta function.

$\varepsilon=\frac{\gamma}{\rho C_{E}}:$ Dimensionless mechanical coupling constant

$\eta$ : The entropy density.

$\zeta=\frac{\rho C_{E}}{k}:$ The thermal viscosity.

$C=\frac{\rho C_{E}}{T_{o}}$

$\theta=\left(T-T_{o}\right):$ The dynamical temperature increment such that $\frac{\left|T-T_{o}\right|}{T_{o}} \ll 1$.

$\lambda, \mu$ : Lamé's constants.

$\rho:$ Mass density.

$\sigma_{i j}$ : Components of stress tensor.

$\sigma=\sigma_{x x}$ : The principal stress component.

$\tau_{o}$ : One relaxation time parameter. 